\title{
On collineations and dualities of finite generalized polygons
}

\author{
Beukje Temmermans Joseph A. Thas Hendrik Van Maldeghem* \\ Department of Pure Mathematics and Computer Algebra \\ Ghent University, Krijgslaan 281, S22, B-9000 Gent \\ email: btemmerm@cage.UGent.be, jat@cage.UGent.be, hvm@cage.UGent.be
}

\begin{abstract}
In this paper we generalize a result of Benson to all finite generalized polygons. In particular, given a collineation $\theta$ of a finite generalized polygon $\mathcal{S}$, we obtain a relation between the parameters of $\mathcal{S}$ and, for various natural numbers $i$, the number of points $x$ which are mapped to a point at distance $i$ from $x$ by $\theta$. As a special case we consider generalized $2 n$-gons of order $(1, t)$ and determine, in the generic case, the exact number of absolute points of a given duality of the underlying generalized $n$-gon of order $t$.
\end{abstract}

\section{Introduction}

Given a finite generalized quadrangle of order $(s, t)$, and a collineation $\theta$, there is a connection between the parameters $s, t$, the number $f_{0}$ of fixed points and the number $f_{1}$ of points mapped under $\theta$ to collinear points, given by Benson's theorem [1]:

$$
(1+t) f_{0}+f_{1} \equiv 1+s t \bmod s+t .
$$

The natural question arising here is whether there exists a similar formula for a duality of a generalized quadrangle $\mathcal{S}$ of order $s$. Of course, a duality cannot fix points or lines,

\footnotetext{
*Research supported by the Fund for Scientific Research - Flanders (FWO - Vlaanderen)
} 
but elements can be mapped to elements at distance 1 or 3 . In the former case, we say that these elements are absolute. It will turn out that, in the generic case, i.e., in the case where $2 s$ is not a square, there are always exactly $1+s$ absolute points, for every duality. This yields an alternative proof for the fact that polarities in $\mathcal{S}$ can only exist if $2 s$ is a square (as a geometric argument immediately shows that there must be $1+s^{2}$ absolute points).

More generally, one can ask for similar restrictions on collineations and dualities for an arbitrary finite generalized polygon. This is exactly what we are going to do in the present paper. Roughly speaking, we first generalize Benson's formula to finite generalized hexagons, octagons and dodecagons with parameters $s, t$, and then show how these can be used to obtain new results on dualities in projective planes, generalized quadrangles and hexagons. The extension of Benson's formula has a straightforward part, but there is also a less trivial observation which precisely allows us to draw some rather strong conclusions in the case of dualities, thereby producing new results even for finite projective planes.

The main application of our results lies in the classification of finite generalized polygons whose collineation or duality group satisfies some given transitivity property like flagtransitivity, or sharp transitivity on points or lines. For an explicit application, see [10].

\section{Notation and Main Results}

Throughout, we shall deal with finite generalized polygons of order $(s, t)$. A generalized $n$-gon $\mathcal{S}=(\mathcal{P}, \mathcal{L} \mathrm{I})$ of order $(s, t)$ is a point-line geometry with point set $\mathcal{P}$, line set $\mathcal{L}$ and symmetric incidence relation $I$, such that every line is incident with $1+s$ points, every point with $1+t$ lines, the diameter of the incidence graph (i.e. the graph with vertex set $\mathcal{P} \cup \mathcal{L}$ and adjacency given by $\mathrm{I}$ ) is $n$ and its girth is $2 n$. Here, $n \geq 2$, and for $n=2$, the incidence graph is a complete bipartite graph and hence $\mathcal{S}$ is a trivial geometry in which every point is incident with every line. So in the sequel we will always assume $n \geq 3$.

Note that in a generalized $n$-gon with $n \geq 3$ each line is determined by the set of points it is incident with. So, we can view a generalized $n$-gon $\mathcal{S}=(\mathcal{P}, \mathcal{L}, \mathrm{I})$ as the set of points $\mathcal{P}$ endowed with a set $\mathcal{B}$ of subsets of $\mathcal{P}$, where $\mathcal{B}=\{B \subseteq \mathcal{P}:(\exists L \in \mathcal{L})(x \in B \Leftrightarrow x I L)\}$. In this case, there is no need for an incidence relation and we denote $\mathcal{S}=(\mathcal{P}, \mathcal{B})$. Related to this point of view is the point graph of $\mathcal{S}$, which is the graph with vertex set $\mathcal{P}$ and adjacency given by collinearity (two points $x, y$ are collinear, in symbols $x \sim y$, if they are distinct and incident with a common line). We will denote the distance function in the point graph by $\mathrm{d}$. 
If $\mathcal{S}=(\mathcal{P}, \mathcal{L}, \mathrm{I})$ is a generalized polygon of order $(s, t)$, then $\mathcal{S}^{\text {dual }}=(\mathcal{L}, \mathcal{P}, \mathrm{I})$ is also a generalized polygon, now of order $(t, s)$, called the dual of $\mathcal{S}$. This gives rise the principle of duality, which states that every theorem in the theory of generalized polygons can be dualized by interchanging the roles of $\mathcal{P}$ and $\mathcal{L}$, and of $s$ and $t$. If $s=t$, then we say that $\mathcal{S}$ has order $s$.

Generalized 3-gons of order $(s, t)$ only exist for $s=t$ and for $s \geq 2$ they are exactly the projective planes of order $s=t$. Generalized 4-gons are usually called generalized quadrangles and these structures have been investigated in detail; see [9]. Generalized 6gons, 8-gons and 12-gons are also called generalized hexagons, octagons and dodecagons, respectively. We refer to [12] and [15] for extensive surveys on (finite and infinite) generalized polygons. We content ourselves here with mentioning that generalized polygons were introduced by Jacques Tits [13] in 1959. They are the spherical buildings of rank 2 . As such they provide the natural geometries for Chevalley groups of rank 2, and, more generally, for algebraic, classical and mixed groups, and twisted versions, all of relative rank 2.

A major result on finite generalized polygons is due to Feit \& Higman [6] and states that for a finite generalized $n$-gon, $n \geq 3$, of order $(s, t)$, we always have either $n=3$ (and then $s=t$ ), or $n=4$ (and then $s+t$ divides $s t(1+s t)$ ), or $n=6$ (and then $s t$ is a perfect square), or $n=8$ (and then $2 s t$ is a perfect square), or $n=12$ (and then $1 \in\{s, t\}$ ). Each generalized $n$-gon $\mathcal{S}=(\mathcal{P}, \mathcal{L}, \mathrm{I})$ of order $(s, s)$ gives rise to a unique generalized $2 n$-gon $2 \mathcal{S}=(\mathcal{P} \cup \mathcal{L}, \mathcal{E})$ of order $(1, s)$, called the double of $\mathcal{S}$, where $\mathcal{E}$ is the set of flags of $\mathcal{S}$ (and a flag is an incident point-line pair). This construction has an inverse which is unique up to duality (see [14], cp. [15], Theorem 1.6.2).

A collineation $\theta$ of a generalized polygon $\mathcal{S}=(\mathcal{P}, \mathcal{B})$ is a permutation of $\mathcal{P}$ that induces a permutation of $\mathcal{B}$. If $\mathcal{S}^{\prime}=\left(\mathcal{P}^{\prime}, \mathcal{B}^{\prime}\right)$ is a second generalized polygon, then an isomorphism $\sigma: \mathcal{S} \rightarrow \mathcal{S}^{\prime}$ is a bijection from $\mathcal{P}$ to $\mathcal{P}^{\prime}$ that induces a bijection from $\mathcal{B}$ to $\mathcal{B}^{\prime}$. A duality of $\mathcal{S}$ is an isomorphism from $\mathcal{S}$ to $\mathcal{S}^{\text {dual }}$. In this case we call $\mathcal{S}$ self-dual. Note that for a self-dual generalized polygon of order $(s, t)$ necessarily holds that $s=t$.

If $\theta$ is a duality of $\mathcal{S}$, then an absolute element for $\theta$ is a point or a line incident with its image.

Now let $\theta$ be a collineation of a finite generalized $n$-gon $\mathcal{S}=(\mathcal{P}, \mathcal{L}, \mathrm{I})$ with parameters $s, t$. Let $f_{i}, 0 \leq i \leq \frac{n}{2}$, be the number of points $x$ of $\mathcal{S}$ that are mapped under $\theta$ onto a point at distance $i$ from $x$, measured in the point graph (or, equivalently, distance $2 i$ in the incidence graph). Also, the point graph has one eigenvalue (namely, $s(1+t)$ ) with multiplicity 1 , it has always an eigenvalue equal to $-1-t$ and it has $\frac{n}{2}-1$ eigenvalues different from $-1-t$ with multiplicity $>1$. We denote the latter with $\stackrel{\xi}{j}_{j}, 1 \leq j \leq \frac{n}{2}-1$. 
Benson's theorem [1] says that, if $n=4$, then $(1+t) f_{0}+f_{1} \equiv 1+s t \bmod s+t$. Equivalently, there exists an integer $k_{1}$ such that $(1+s)(1+t)+k_{1}(s+t)=(1+t) f_{0}+f_{1}$. Our Main Result generalizes this equivalent formulation as follows:

Main Result With the above notation, for $n \in\{4,6,8,12\}$, there exist integers $k_{j}$, $1 \leq j \leq \frac{n}{2}-1$, and, for each $k \in\left\{1,2, \ldots, \frac{n}{2}-1\right\}$, explicitly defined polynomial expressions $P_{k, i}(s, t), 0 \leq i \leq k-1$, in $s$ and $t$, such that

$$
(1+s)^{k}(1+t)^{k}+\sum_{j=1}^{n / 2-1} k_{j}\left(\xi_{j}+1+t\right)^{k}=f_{k}+\sum_{i=0}^{k-1} P_{k, i}(s, t) f_{i} .
$$

We explicitly determine the expressions $P_{k, i}$ in this theorem below, for each $n$ separately: for $n=4$, see Theorem 4.1 (this is Benson's theorem); for $n=6$, see Theorems 5.1 and 5.4; for $n=8$, see Theorems 6.1, 6.4 and 6.5; for $n=12$, see Theorems 7.1, 7.2, 7.3, 7.4 and 7.5.

Although the expressions are, especially for the cases $n=8,12$, rather involved and cumbersome, we are able to draw some interesting conclusions. In particular we obtain strong restrictions on the number of absolute elements of a duality. Generically, we obtain the following result.

Main Corollary Let $\theta$ be a duality of a finite generalized n-gon with parameter $s$. Then there is at least one absolute point for $\theta$. Suppose now that $s$ is not a square if $n=3$, that $2 s$ is not a square if $n=4$, and that none of $s$ and $3 s$ are squares if $n=6$. Then there are exactly $1+s$ absolute points for $\theta$.

Much more precise information is contained in the corollaries below. The consequences of our Main Result seem endless, and we have included only a few of them. They are related to ovoids, subpolygons, involutions and dualities.

The paper is organized as follows. In Section 3, we prove some general statements about eigenvalues and multiplicities, and recall a useful result concerning the adjacency matrix of a generalized polygon. In Section 4 we repeat Benson's theorem and write down some consequences, as a warming-up for the more general cases treated in Section 5 (generalized hexagons), Section 6 (generalized octagons) and Section 7 (generalized dodecagons).

\section{Some general observations}

We will use the following notation. Suppose that $\mathcal{S}$ is a finite generalized polygon of order $(s, t)$. Let $v$ be the number of points of $\mathcal{S}$ and $b$ the number of lines. Put $\mathcal{P}=\left\{x_{i}: 1 \leq i \leq\right.$ 
$v\}$ and $\mathcal{L}=\left\{L_{j}: 1 \leq j \leq b\right\}$. Let $D$ be an incidence matrix of $\mathcal{S}$, i.e., the rows of $D$ are labelled by the points of $\mathcal{S}$, the columns by the lines of $\mathcal{S}$ and the $(x, L)$ )-entry of $D$ (where $x \in \mathcal{P}$ and $L \in \mathcal{L})$ is equal to 1 if $x \mathrm{I} L$; otherwise it is 0 . Then $M:=D D^{T}=A+(t+1) I$, where $A$ is an adjacency matrix of the point graph of $\mathcal{S}$. Let $\theta$ be an automorphism of $\mathcal{S}$ of order $n$ and let $Q=\left(q_{i j}\right)$ be the $v \times v$ matrix with $q_{i j}=1$ if $x_{i}^{\theta}=x_{j}$ and $q_{i j}=0$ otherwise (in fact $Q$ is the permutation matrix belonging to $\theta$ with respect to the action on $\mathcal{P}$ ). Similarly, let $R=\left(r_{i j}\right)$ be the permutation matrix belonging to $\theta$ with respect to the action of $\theta$ on $\mathcal{L}$ (so $r_{i j}=1$ if $L_{i}^{\theta}=L_{j}$ and $r_{i j}=0$ otherwise). Then $D R=Q D$. Because $Q$ and $R$ are permutation matrices, it follows that $Q^{T}=Q^{-1}$ and $R^{T}=R^{-1}$, so we have $Q M=Q D D^{T}=D R D^{T}=D R R^{T} D^{T}\left(Q^{-1}\right)^{T}=D D^{T} Q=M Q$. Hence $Q M=M Q$. Because $n$ is the order of $\theta$ and $Q M=M Q$, we have $(Q M)^{n}=Q^{n} M^{n}=M^{n}$. It follows that the eigenvalues of $Q M$ are of the form $\xi \lambda$ with $\lambda$ an eigenvalue of $M$ and $\xi$ an $n^{\text {th }}$ root of unity. Note that the eigenvalue $(1+s)(1+t)$ of $M$ is also an eigenvalue of $Q M$ with multiplicity 1.

Lemma 3.1 Suppose that $\xi$ and $\xi^{\prime}$ are both primitive $d^{\text {th }}$ roots of unity, with $d$ a divisor of $n$, and let $\lambda$ be an eigenvalue of $M$. If at least one of $\xi \lambda$ and $\xi^{\prime} \lambda$ is an eigenvalue of $Q M$, than they both are and they have the same multiplicity.

Proof. The coefficients of the characteristic polynomial of $Q M$ are integers. The minimal polynomials (over $\mathbb{Q}$ ) of $\xi \lambda$ and $\xi^{\prime} \lambda$ coincide, hence $\xi \lambda$ and $\xi^{\prime} \lambda$ have the same multiplicity.

Lemma 3.2 Let $\xi$ be an $n^{\text {th }}$ root of unity and $\lambda$ an eigenvalue of $M$. Then the multiplicity of $\xi \lambda$ as an eigenvalue of $Q M$ is equal to the multiplicity of $\xi \lambda^{j}$ as an eigenvalue of $Q M^{j}$, with $j=2,3, \cdots$.

Proof. We first claim that there exists a basis of eigenvectors with eigenvalues $\xi \lambda$ for $Q M$ (with $\xi$ ranging over the $n^{\text {th }}$ roots of unity) in the eigenspace $V$ which corresponds with the eigenvalue $\lambda$ for $M$. First note that, if $\vec{v} \in V$, then

$$
\begin{aligned}
M Q \vec{v} & =Q M \vec{v} \\
& =Q \lambda \vec{v} \\
& =\lambda Q \vec{v},
\end{aligned}
$$

and hence $Q \vec{v} \in V$. So $Q$ preserves $V$ and induces an isometry in $V, V$ viewed as the standard Euclidian space. Hence there exists an orthonormal basis $B$ of $V$ of eigenvectors 
of $Q$. Since $Q^{n}=1$, each eigenvalue $\xi$ is an $n^{\text {th }}$ root of unity. If $\vec{v} \in B$ is an eigenvector for $Q$ with eigenvalue $\xi$, then $M Q \vec{v}=\xi \lambda \vec{v}$. This shows our claim.

Now we will prove that the multiplicity of $\xi \lambda$ for $Q M$ is equal to the multiplicity of $\xi \lambda^{j}$ for $Q M^{j}$, which is the dimension of the eigenspace which corresponds with this eigenvalue. If $\vec{v}$ is an eigenvector of $Q M$ with eigenvalue $\xi \lambda$, then we have

$$
\begin{aligned}
Q M^{j} \vec{v} & =M^{j-1}(Q M \vec{v}) \\
& =M^{j-1} \xi \lambda \vec{v} \\
& =\xi \lambda M^{j-1} \vec{v} \\
& =\xi \lambda^{j} \vec{v} .
\end{aligned}
$$

Hence $\vec{v}$ is an eigenvector of $Q M^{j}$ with eigenvalue $\xi \lambda^{j}$. So the eigenvectors of $Q M$ with eigenvalue $\xi \lambda$ form a subset of the eigenvectors of $Q M^{j}$ with eigenvalue $\xi \lambda^{j}$. Hence, since $Q M$, and similarly also $Q M^{j}$, is diagonalizable,

$$
\begin{gathered}
v=\sum_{m=1}^{n} \text { dimension of eigenspace corresponding with eigenvalue } e^{\frac{m 2 \pi i}{n}} \lambda^{j} \text { of } Q M^{j} \\
\geq \sum_{m=1}^{n} \text { dimension of eigenspace corresponding with eigenvalue } e^{\frac{m 2 \pi i}{n}} \lambda \text { of } Q M=v .
\end{gathered}
$$

Hence every two corresponding terms in these sums must be equal.

In the sequel, we will also consider thin generalized polygons, by which we mean generalized polygons $\mathcal{S}$ with either $s=1$ or $t=1$. As already noted, a generalized $2 n$-gon $\mathcal{S}$ of order $(1, t)$ is the double $2 \mathcal{S}^{\prime}$ of a - up to duality uniquely — defined generalized $n$-gon $\mathcal{S}^{\prime}$ of order $t$. Every collineation of $\mathcal{S}$ induces either a unique collineation of $\mathcal{S}^{\prime}$ or a unique duality of $\mathcal{S}^{\prime}$. In the first case a point $x$ of $\mathcal{S}$ is mapped onto a point at even distance from $x$; in the second case a point $x$ of $\mathcal{S}$ is mapped onto a point at odd distance from $x$. In the sequel we will call $\mathcal{S}^{\prime}$ the underlying generalized $n$-gon.

The following lemma is well known, e.g. see [2].

Lemma 3.3 Let $A$ be the adjacency-matrix of a generalized $2 n$-gon of order $(s, t)$. Since for two points $x$ and $y$, the $(x, y)$-entry only depends on the distance $2 i$ (in the incidence graph) between $x$ and $y$, the same is true for $A^{k}$ with $k \geq 1$. Hence we can denote this entry by $a_{i}^{(k)}$. Let $p_{j}^{i}$ be the number of points at distance $2 j$ from $x$ and collinear to $y$, with $x$ and $y$ as above. Then $a_{i}^{(k+1)}=\sum_{j=0}^{n} p_{j}^{i} a_{j}^{(k)}$, with $i \in\{0,1, \cdots, n\}$. 
For a generalized $n$-gon $p_{j}^{i}$ is equal to:

$$
\begin{cases}s(t+1) & \text { if } j=1, i=0 \\ 1 & \text { if } 0 \leq j=i-1 \leq n-2 \\ s-1 & \text { if } 1 \leq j=i \leq n-1 \\ s t & \text { if } 2 \leq j=i+1 \leq n \\ t+1 & \text { if } j=n-1, i=n \\ (s-1)(1+t) & \text { if } j=i=n \\ 0 & \text { otherwise. }\end{cases}
$$

We end with a straightforward observation.

Lemma 3.4 A duality $\theta$ of a generalized polygon has as many absolute points as absolute lines.

Proof. This follows from the fact that, if $x \mathrm{I} x^{\theta}$, then $x^{\theta} \mathrm{I}\left(x^{\theta}\right)^{\theta}$, which implies that, if $x$ is an absolute point, then $x^{\theta}$ is an absolute line. It is now easy to see that $\theta$ induces a bijection from the set of absolute points to the set of absolute lines.

\section{Collineations of generalized quadrangles}

At first we will have a look at a generalized quadrangle $\mathcal{S}=(\mathcal{P}, \mathcal{L}, \mathrm{I})$ of order $(s, t)$. Let $D, M, A, Q$ and $\theta$ be defined as before. If $M=A+(t+1) I$, then $M$ has eigenvalues $\tau_{0}=(1+s)(1+t), \tau_{1}=0$ and $\tau_{2}=s+t$, with respective multiplicities $m_{0}=1$, $m_{1}=s^{2}(1+s t) /(s+t)$ and $m_{2}=s t(1+s)(1+t) /(s+t)$ (cf. Table 6.4 in [2]). Now we have the following theorem.

Theorem 4.1 (Benson [1]) If $f_{0}$ is the number of points fixed by the automorphism $\theta$ and if $f_{1}$ is the number of points $x$ for which $x^{\theta} \neq x \sim x^{\theta}$, then

$$
\operatorname{tr}(Q M)=(1+t) f_{0}+f_{1} \quad \text { and } \quad(1+t) f_{0}+f_{1} \equiv 1+s t(\bmod s+t) .
$$

Proof. For the proof of this theorem, we refer to [1].

Remark 4.2 We can also write the conclusion of Theorem 4.1 as follows: 


$$
\operatorname{tr}(Q M)=(1+t) f_{0}+f_{1}=k(s+t)+(1+s)(1+t)
$$

Corollary 4.3 Let $\mathcal{S}$ be a generalized quadrangle of order $(s, t)$ and let $\theta$ be an automorphism of $\mathcal{S}$. Suppose that $s$ and $t$ are not relatively prime, then there exists at least one fixpoint or at least one point which is mapped to a point collinear to itself.

Proof. Suppose that there are no fixpoints and no points which are mapped to a collinear point. Then $f_{0}=f_{1}=0$. Because of the previous theorem $1+s t+l(s+t)$ has to be 0 for some $l$, or $s t+l(s+t)=-1$. But because $s$ and $t$ are not relatively prime, there exists an integer $m>1$ which divides both $s$ and $t$. Hence $m$ divides $s t+l(s+t)$. But $m$ does not divide -1 and we have a contradiction.

Corollary 4.4 Suppose that $\mathcal{S}$ is a generalized quadrangle of order $s$ and $\theta$ is a nontrivial automorphism of $\mathcal{S}$. If $s$ is even, then $\theta$ cannot fix any ovoid pointwise.

Proof. Suppose that $\mathcal{O}$ is an ovoid which is fixed pointwise by $\theta$. Then (with the notation of the previous theorem) $f_{0}=1+s^{2}=|\mathcal{O}|$. Note that by 2.4.1 in [9] $\theta$ cannot fix anything else. Suppose that there exists a point $x$ which is mapped to a collinear point by $\theta$. Take a line through $x$ different from $x x^{\theta}$. This line contains a point $y$ of the ovoid. Now the line $x y$ is mapped to the line $x^{\theta} y$, hence we have a triangle which is a contradiction. So $f_{1}=0$. Because of Benson's theorem it follows that $(1+s)\left(1+s^{2}\right) \equiv 1+s^{2}(\bmod 2 s)$ or $s\left(1+s^{2}\right) \equiv 0(\bmod 2 s)$. Hence $1+s^{2}$ is even and so $s$ has to be odd.

Let $\mathrm{Q}(4, q)$ be a nonsingular (parabolic) quadric in the projective space $\mathrm{PG}(4, q)$, and let $H$ be a hyperplane of $\mathrm{PG}(4, q)$ meeting $\mathrm{Q}(4, q)$ in a nonsingular elliptic quadric $\mathrm{Q}^{-}(3, q)$. If $q$ is odd, then $H$ has a pole $x$ (the intersection of all hyperplanes tangent to $\mathrm{Q}(4, q)$ at points of $\left.\mathrm{Q}^{-}(3, q)\right)$, and the unique involutive perspectivity of $\mathrm{PG}(4, q)$ with center $x$ and axis $H$ induces a nontrivial collineation in the generalized quadrangle $Q(4, q)$ fixing the ovoid $\mathrm{Q}^{-}(3, q)$ pointwise. Hence Corollary 4.4 is not valid for $s$ odd.

Corollary 4.5 Suppose that $\mathcal{S}$ is a generalized quadrangle of order $s$ and $\theta$ is a nontrivial automorphism of $\mathcal{S}$. If $s$ is even, then $\theta$ cannot fix any thin subquadrangle of order $(1, s)$ pointwise.

Proof. Suppose that $\mathcal{S}^{\prime}$ is a thin subquadrangle of $\mathcal{S}$ of order $(1, s)$ which is fixed pointwise by $\theta$. Then we claim $f_{0}=2(s+1)=\left|\mathcal{S}^{\prime}\right|$. Indeed, if $\theta$ fixes a point not belonging to $\mathcal{S}^{\prime}$, then $\theta$ would fix a subquadrangle $\mathcal{S}^{\prime \prime}$ of order $\left(s^{\prime \prime}, s\right)$ with $\mathcal{S}^{\prime} \subset \mathcal{S}^{\prime \prime} \subseteq \mathcal{S}$. By 2.2.1 of [9] it 
follows that $s \geq s^{\prime \prime} s$. Hence $s^{\prime \prime}=1$ a contradiction. Now suppose that $x$ is a point of $\mathcal{S}^{\prime}$. There are $s+1$ lines through $x$ in $\mathcal{S}^{\prime}$ and on each of these lines there is precisely one other point which belongs to $\mathcal{S}^{\prime}$. Take such a point $y$. On the line $x y$ of $\mathcal{S}$ there are $s-1$ other points which are mapped to each other (not fixed). So we already have $(s-1)(s+1)^{2}$ points which are mapped to a collinear point by $\theta$. Suppose that there is a point $z$ of $\mathcal{S}$ which does not lie on a line of $\mathcal{S}^{\prime}$ and which is mapped to a point collinear to itself. Then by 2.2.1 of [9] the line $z z^{\theta}$ intersects a line of $\mathcal{S}^{\prime}$ (which is fixed under $\theta$ ). But then the line $z z^{\theta}$ also has to be fixed and we obtain a contradiction. Hence $f_{1}=(s-1)(s+1)^{2}$. Now Theorem 4.1 implies that there exists an integer $k$ with

$$
k(2 s)+(1+s)^{2}=2(1+s)^{2}+(s-1)(s+1)^{2} .
$$

Hence $k=\frac{(s+1)^{2}}{2}$ and so $s$ is odd.

\section{Collineations of hexagons and dualities of projec- tive planes}

Next we will generalize Benson's theorem for hexagons.

Suppose that $\mathcal{S}$ is a generalized hexagon. Let $\theta$ be an automorphism of $\mathcal{S}$ and let $f_{0}$ be the number of fixpoints, $f_{1}$ the number of points which are mapped on a collinear point $\left(\mathrm{d}\left(x^{\theta}, x\right)=1\right.$ in the point graph of $\left.\mathcal{S}\right)$ and $f_{2}$ the number of points which are mapped on a point at distance 2 from itself (in the point graph). The matrix $M$ is again equal to $A+(t+1) I$, with $A$ an adjacency matrix of the point graph of $\mathcal{S}$. And $Q$ is the matrix with $q_{i j}=1$ if $x_{i}^{\theta}=x_{j}$ and $q_{i j}=0$ otherwise.

We start from the eigenvalues of $A$ which are $-1-t, s(t+1),-1+s+\sqrt{s t}$ and $-1+s-\sqrt{s t}$, with respective multiplicities $m_{0}, m_{1}=1, m_{2}$ and $m_{3}$. Because $M=A+(t+1) I$ the eigenvalues of $M$ are as follows (cf. Table 6.4 in [2]). 


\begin{tabular}{|c|c|}
\hline eigenvalues of $M$ & multiplicity \\
\hline 0 & $m_{0}=\frac{s^{3}\left(1+s t+s^{2} t^{2}\right)}{s^{2}+s t+t^{2}}$ \\
$(s+1)(t+1)$ & $m_{1}=1$ \\
$s+t+\sqrt{s t}$ & $m_{2}=\frac{(1+t) s t(1+s)\left(1+s t+s^{2} t^{2}\right)}{2\left(s(t-1)^{2}+t(s-1)^{2}+3 s t+(s-1)(t-1) \sqrt{s t}\right)}$ \\
$s+t-\sqrt{s t}$ & $m_{3}=\frac{(1+t) s t(1+s)\left(1+s t+s^{2} t^{2}\right)}{2\left(s(t-1)^{2}+t(s-1)^{2}+3 s t-(s-1)(t-1) \sqrt{s t}\right)}$ \\
\hline
\end{tabular}

We now have the following result.

Theorem 5.1 Let $\mathcal{S}$ be a generalized hexagon of order $(s, t)$ and let $\theta$ be an automorphism of $\mathcal{S}$. If $f_{i}, i=0,1$, is defined as above, then for some integers $k_{1}$ and $k_{2}$ there holds

$$
k_{1}(s+t+\sqrt{s t})+k_{2}(s+t-\sqrt{s t})+(1+s)(1+t)=(1+t) f_{0}+f_{1} .
$$

Proof. Suppose that $\theta$ has order $n$, so that $(Q M)^{n}=Q^{n} M^{n}=M^{n}$. It follows that the eigenvalues of $Q M$ are the eigenvalues of $M$ multiplied by the appropriate roots of unity. Let $J$ be the $v \times v$ matrix with all entries equal to 1 . Since $M J=(1+s)(1+t) J$, we have $(Q M) J=(1+s)(1+t) J$, so $(1+s)(1+t)$ is an eigenvalue of $Q M$. Because $m_{1}=1$, it follows that this eigenvalue of $Q M$ has multiplicity 1. Further it is clear that 0 is an eigenvalue of $Q M$ with multiplicity $m_{0}$. For each divisor $d$ of $n$, let $\xi_{d}$ denote a primitive $d^{\text {th }}$ root of unity, and put $U_{d}=\sum \xi_{d}^{i}$, where the summation is over those integers $i \in\{1,2, \ldots, d-1\}$ that are relatively prime to $d$. Now $U_{d}$ is the coefficient of the term of the second largest degree of the corresponding cyclotomic polynomial $\Phi_{n}(x)$. And since $\Phi_{n}(x) \in \mathbb{Z}[x]$, by [7], $U_{d}$ is an integer. For each divisor $d$ of $n$, the primitive $d^{\text {th }}$ roots of unity all contribute the same number of times to the eigenvalues $\varphi$ of $Q M$ with $|\varphi|=s+t+\sqrt{s t}$ and also the primitive $d^{\text {th }}$ roots of unity all contribute the same number of times to the eigenvalues $\varphi^{\prime}$ of $Q M$ with $\left|\varphi^{\prime}\right|=s+t-\sqrt{s t}$, because of Lemma 3.1. Let $a_{d}$ denote the multiplicity of $\xi_{d}(s+t+\sqrt{s t})$ and let $b_{d}$ denote the multiplicity of $\xi_{d}(s+t-\sqrt{s t})$ as eigenvalues of $Q M$, with $d \mid n$ and $\xi_{d}$ a primitive $d^{\text {th }}$ root of unity. Then we have:

$$
\operatorname{tr}(Q M)=\sum_{d \mid n} a_{d}(s+t+\sqrt{s t}) U_{d}+\sum_{d \mid n} b_{d}(s+t-\sqrt{s t}) U_{d}+(1+s)(1+t)
$$


Or

$$
\operatorname{tr}(Q M)=k_{1}(s+t+\sqrt{s t})+k_{2}(s+t-\sqrt{s t})+(1+s)(1+t)
$$

with $k_{1}$ and $k_{2}$ integers.

Since the entry on the $i^{\text {th }}$ row and $i^{\text {th }}$ column of $Q M$ is the number of lines incident with $x_{i}$ and $x_{i}^{\theta}$, we have $\operatorname{tr}(Q M)=(1+t) f_{0}+f_{1}$. Hence

$$
k_{1}(s+t+\sqrt{s t})+k_{2}(s+t-\sqrt{s t})+(1+s)(1+t)=(1+t) f_{0}+f_{1},
$$

with $k_{1}$ and $k_{2}$ integers.

The following corollary is the analogue of Corollary 4.3

Corollary 5.2 Let $\mathcal{S}$ be a generalized hexagon of order $(s, t)$ and let $\theta$ be an automorphism of $\mathcal{S}$. If $s$ and $t$ are not relatively prime, then there exists at least one fixpoint or at least one point which is mapped to a point collinear to itself.

Proof. Suppose that there are no fixpoints and no points which are mapped to a collinear point. Then $f_{0}=f_{1}=0$. Because of the previous theorem, $k_{1}(s+t+\sqrt{s t})+k_{2}(s+t-\sqrt{s t})+$ $(1+s)(1+t)$ has to be equal to 0 . Hence $k_{1}(s+t+\sqrt{s t})+k_{2}(s+t-\sqrt{s t})+s+t+s t=-1$. But because $s$ and $t$ are not relatively prime, there exists an integer $m>1$ which divides $s$ and $t$. Hence $m$ divides $k_{1}(s+t+\sqrt{s t})+k_{2}(s+t-\sqrt{s t})+s+t+s t$, but $m$ does not divide -1 and we have a contradiction.

Corollary 5.3 Let $\mathcal{S}$ be a generalized hexagon of order $(s, t)$ and let $\theta$ be an involution of $\mathcal{S}$. If $s$ and $t$ are not relatively prime, then there exists at least one fixpoint or at least one fixline.

Proof. This follows immediately from the previous corollary because if there is a point $x$ which is mapped to a point collinear to $x$ by the involution $\theta$, then the line $x x^{\theta}$ is a fixline.

Now we have a look at the formula in Theorem 5.1 in the special case of a thin hexagon $(s=1)$. Then we have:

$$
k_{1}(1+t+\sqrt{t})+k_{2}(1+t-\sqrt{t})+2(1+t)=(1+t) f_{0}+f_{1} .
$$


If $t$ is not a square, then it follows that $k_{1}=k_{2}$, hence we obtain $k_{1} 2(t+1)+2(t+1)=$ $(t+1) f_{0}+f_{1}$ and so $t+1$ has to divide $f_{1}$. But we will improve this below (see Corollary 5.6). Note that either $f_{0}$ or $f_{1}$ is 0 , according to whether the corresponding collineation $\theta$ of $\mathcal{S}$ induces a duality or a collineation in the underlying projective plane.

The method exploited in the proof of Theorem 5.1 is completely similar to the original approach of Benson. However, in order to be able to say more, an additional idea is needed. Motivated by Lemma 3.2, our idea is now to apply Benson's approach to the matrix $M^{2}$ (which, in case of generalized quadrangles, does not give anything new). Without Lemma 3.2, this would not give too much, but in combination with that lemma, we will obtain new and quite interesting results, even for the case of projective planes!

Theorem 5.4 Let $\mathcal{S}$ be a generalized hexagon of order $(s, t)$ and let $\theta$ be an automorphism of $\mathcal{S}$. If $f_{i}, i=0,1,2$, is as before, then for the integers $k_{1}$ and $k_{2}$ of Theorem 5.1 there holds

$k_{1}(s+t+\sqrt{s t})^{2}+k_{2}(s+t-\sqrt{s t})^{2}+((1+s)(1+t))^{2}=(1+s+t)(1+t) f_{0}+(1+s+2 t) f_{1}+f_{2}$.

Proof. Suppose that $M, A$ and $Q$ are defined as before. Suppose that $\theta$ has order $n$, so that $\left(Q M^{2}\right)^{n}=Q^{n} M^{2 n}=M^{2 n}$. It follows that the eigenvalues of $Q M^{2}$ are the eigenvalues of $M^{2}$ multiplied by the appropriate roots of unity. Since $M^{2} J=((1+s)(1+t))^{2} J$, we have $\left(Q M^{2}\right) J=((1+s)(1+t))^{2} J$, so $((1+s)(1+t))^{2}$ is an eigenvalue of $Q M^{2}$. By Lemma 3.2 and the proof of Theorem 5.1 it follows that this eigenvalue of $Q M^{2}$ has multiplicity 1. Further it is clear that 0 is an eigenvalue of $Q M^{2}$ with multiplicity $m_{0}$. For each divisor $d$ of $n$, let $\xi_{d}$ again denote a primitive $d^{\text {th }}$ root of unity, and put $U_{d}=\sum \xi_{d}^{i}$, where the summation is over those integers $i \in\{1,2, \ldots, d-1\}$ that are relatively prime to $d$. Then $U_{d}$ is an integer [7]. For each divisor $d$ of $n$, the primitive $d^{\text {th }}$ roots of unity all contribute the same number of times to the eigenvalues $\varphi$ of $Q M^{2}$ with $|\varphi|=(s+t+\sqrt{s t})^{2}$ and also the primitive $d^{\text {th }}$ roots of unity all contribute the same number of times to the eigenvalues $\varphi^{\prime}$ of $Q M^{2}$ with $\left|\varphi^{\prime}\right|=(s+t-\sqrt{s t})^{2}$, because of Lemma 3.1. Let $a_{d}$ denote the multiplicity of $\xi_{d}(s+t+\sqrt{s t})^{2}$ and let $b_{d}$ denote the multiplicity of $\xi_{d}(s+t-\sqrt{s t})^{2}$ as eigenvalues of $Q M^{2}$, with $d \mid n$ and $\xi_{d}$ a primitive $d^{\text {th }}$ root of unity. Then we have:

$$
\operatorname{tr}\left(Q M^{2}\right)=\sum_{d \mid n} a_{d}(s+t+\sqrt{s t})^{2} U_{d}+\sum_{d \mid n} b_{d}(s+t-\sqrt{s t})^{2} U_{d}+((1+s)(1+t))^{2}
$$

or

$$
\operatorname{tr}\left(Q M^{2}\right)=k_{1}(s+t+\sqrt{s t})^{2}+k_{2}(s+t-\sqrt{s t})^{2}+((1+s)(1+t))^{2}
$$


with $k_{1}$ and $k_{2}$ integers. Clearly we have $\operatorname{tr}(Q A)=f_{1}$.

The matrix $A^{2}=\left(a_{i j}\right)$ is the matrix with $s(1+t)$ along the main diagonal and on the other entries we have $a_{i j}=s-1$ if $x_{i} \sim x_{j}, a_{i j}=1$ if $\mathrm{d}\left(x_{i}, x_{j}\right)=2$ and $a_{i j}=0$ otherwise. Hence $\operatorname{tr}\left(Q A^{2}\right)=s(1+t) f_{0}+(s-1) f_{1}+f_{2}$. It follows that

$$
\begin{aligned}
& \operatorname{tr}\left(Q M^{2}\right) \\
= & \operatorname{tr}\left(Q(A+(1+t) I)^{2}\right) \\
= & \operatorname{tr}\left(Q A^{2}\right)+2(1+t) \operatorname{tr}(Q A)+(1+t)^{2} \operatorname{tr}(Q) \\
= & s(1+t) f_{0}+(s-1) f_{1}+f_{2}+2(1+t) f_{1}+(1+t)^{2} f_{0} \\
= & (1+s+t)(1+t) f_{0}+(1+s+2 t) f_{1}+f_{2} .
\end{aligned}
$$

Finally, the integers $k_{1}$ and $k_{2}$ are the same integers as in Theorem 5.1 by Lemma 3.2. This completes the proof of the theorem.

Corollary 5.5 Suppose that we have a thin hexagon of order $(1, t)$, with $t \neq 1$. Consider a duality in the underlying projective plane. If $t$ is not a square, then $f_{1}=2(1+t)$. If $t$ is a square, then $f_{1} \equiv 2 \bmod 2 \sqrt{t}$. In particular there is at least one absolute line and one absolute point.

Proof. Since we have a duality in the underlying projective plane, we know that $f_{0}=0$ and $f_{2}=0$. Because of Theorems 5.1 and 5.4, we have the following equations:

$$
\left\{\begin{array}{l}
k_{1}(1+t+\sqrt{t})+k_{2}(1+t-\sqrt{t})+2(1+t)=f_{1} \\
k_{1}(1+t+\sqrt{t})^{2}+k_{2}(1+t-\sqrt{t})^{2}+(2(1+t))^{2}=(2+2 t) f_{1},
\end{array}\right.
$$

hence

$$
\left\{\begin{array}{l}
k_{1}=\frac{f_{1}-2(1+t)}{2 \sqrt{t}} \\
k_{2}=-\frac{f_{1}-2(1+t)}{2 \sqrt{t}} .
\end{array}\right.
$$

So $\frac{f_{1}-2(1+t)}{2 \sqrt{t}}$ has to be an integer. In the case that $t$ is not a square, this only holds if $f_{1}-2(1+t)=0$. Hence $f_{1}=2(1+t)$ if $t$ is not a square. If $t$ is a square, then $f_{1}-2$ has to be a multiple of $2 \sqrt{t}$. Hence $f_{1} \equiv 2 \bmod 2 \sqrt{t}$.

In view of Lemma 3.4, this immediately implies:

Corollary 5.6 Suppose that $\theta$ is a duality of a projective plane of order $t$, then it has $1+t$ absolute points and $1+t$ absolute lines if $t$ is not a perfect square, and it has 1 $\bmod \sqrt{t}$ absolute points and just as many absolute lines if $t$ is a perfect square. 
If $t$ is a square, then the lower bound of this corollary can be obtained. Indeed, let $b$ be an element of the finite (Galois) field $\mathbb{F}_{t}$ (of $t$ elements) not belonging to the subfield $\mathbb{F}_{\sqrt{t}}$. With the usual representation of the Desarguesian projective plane $\mathrm{PG}(2, t)$ of order $t$ by means of triples of elements of $\mathbb{F}_{t}$ (with round brackets to denote points and square brackets for lines), the map

$$
\theta: \mathrm{PG}(2, t) \rightarrow \mathrm{PG}(2, t):\left\{\begin{aligned}
(x, y, z) & \mapsto\left[x^{\sqrt{t}}, z^{\sqrt{t}}, y^{\sqrt{t}}-b z^{\sqrt{t}}\right] \\
{[u, v, w] } & \mapsto\left(u^{\sqrt{t}}, w^{\sqrt{t}}+b v^{\sqrt{t}}, v^{\sqrt{t}}\right)
\end{aligned}\right.
$$

is a duality. A point $(x, y, z)$ is absolute if and only if $(x, y, z)$ is incident with $\left[x^{\sqrt{t}}, z^{\sqrt{t}}, y^{\sqrt{t}}-\right.$ $b z^{\sqrt{t}}$. If $z \neq 0$, then this obviously implies that

$$
b=\frac{x x^{\sqrt{t}}+\left(y z^{\sqrt{t}}+y^{\sqrt{t}} z\right)}{z z^{\sqrt{t}}}
$$

is fixed by the field automorphism $a \mapsto a^{\sqrt{t}}$, and hence belongs to $\mathbb{F}_{\sqrt{t}}$, a contradiction. Hence, if $(x, y, z)$ is absolute, then $z=0$ and so $x x^{\sqrt{t}}=0$. We obtain a unique absolute point $(0,1,0)$. Likewise, $[0,0,1]$ is the unique absolute line.

For the next corollary we need the following lemma.

Note that for a generalized hexagon of order $(s, t)$ with $s \neq t$ no ovoids exist because of $[8]$.

Lemma 5.7 Suppose that $\mathcal{S}$ is a generalized hexagon of order $s$ and $\theta$ is a collineation of $\mathcal{S}$. If $\mathcal{O}$ is an ovoid of $\mathcal{S}$ which is fixed pointwise by $\theta$ and $\theta$ additionally fixes some point $x \notin \mathcal{O}$, then $\theta$ is the identity.

Proof. There exists a unique point of the ovoid which is collinear to $x$, we call this point $y$. Every point $x^{\prime}$ collinear to $x$ which is not incident with the line $x y$ is collinear to a unique point $y^{\prime} \neq y$ of the ovoid. Since both $x$ and $y^{\prime}$ are fixed, also $x^{\prime}$ is fixed. Now take any point $x^{\prime \prime} \notin\{x, y\}$ that is incident with the line $x y$. Take a point $a$ collinear to $x^{\prime \prime}$, not incident with $x y$. This point is collinear to a unique point $b$ of $\mathcal{O}$. Since $b$ is fixed by $\theta$ and the line $x y$ is fixed by $\theta$, also the point $x^{\prime \prime}$ has to be fixed. So every point collinear to $x$ is fixed. Since $\mathcal{O}$ contains a point at distance 6 from $x$ we can apply Theorem $4.4 .2(v)$ in $[15]$ to obtain that $\theta$ is the identity.

It is well known that the dual $\mathrm{H}(q)^{\text {dual }}$ of the split Cayley hexagon $\mathrm{H}(q)$ of order $q$ admits an ovoid stabilized by the subgroup $\mathrm{SU}_{3}(q)$ of $\mathrm{G}_{2}(q)$, and the elements of $\mathrm{SU}_{3}(q)$ fixing the 
ovoid pointwise are exactly the elements of the center of $\mathrm{SU}_{3}(q)$ (see [3]). If $q$ is divisible by 3 , however, this center is trivial and the ovoid does not admit a nontrivial collineation fixing it pointwise. This is a special case of the following more general phenomenon.

Corollary 5.8 Suppose that $\mathcal{S}$ is a generalized hexagon of order $s$ and $\theta$ is a nontrivial automorphism of $\mathcal{S}$. If $s$ is a multiple of 3 , then $\theta$ cannot fix any ovoid pointwise.

Proof. Suppose that $\mathcal{O}$ is an ovoid of $\mathcal{S}$ which is fixed pointwise by $\theta$. Because of the previous lemma there are no other fixpoints. Then $f_{0}=1+s^{3}=|\mathcal{O}|$. Suppose that there exists a point $x$ which is mapped to a collinear point by $\theta$. Suppose that there is a point $y$ of $\mathcal{O}$ which is collinear to $x$ and not collinear to $x^{\theta}$. Then the line $x y$ will be mapped to the line $x^{\theta} y$, hence we have a triangle, so this is not possible. Now there has to be a point $y^{\prime}$ at distance 4 from $x$ which belongs to the ovoid. By a similar reasoning we become a pentagon. Hence we have a contradiction and $f_{1}$ has to be equal to 0 . Because $\theta$ fixes an ovoid every point is mapped to a point at distance at most 4 from it. So $f_{2}$ is the number of points not on the ovoid, hence $f_{2}=\left(1+s^{3}\right)\left(s+s^{2}\right)$. Because of Theorem 5.1 and Theorem 5.4 we have the following equations

$$
\left\{\begin{array}{l}
k_{1} 3 s+k_{2} s+(1+s)^{2}=(1+s)\left(1+s^{3}\right) \\
k_{1} 9 s^{2}+k_{2} s^{2}+(1+s)^{4}=(1+2 s)(1+s)\left(1+s^{3}\right)+\left(1+s^{3}\right)\left(s+s^{2}\right) .
\end{array}\right.
$$

Hence

$$
\left\{\begin{array}{l}
k_{1}=\frac{(1+s)\left(s^{2}-1\right)}{3} \\
k_{2}=0
\end{array}\right.
$$

Because $k_{1}$ has to be an integer, it follows that $s$ cannot be a multiple of 3 .

The split Cayley hexagon $\mathrm{H}(q)$ admits a subhexagon of order $(1, q)$, stabilized by the group $\mathrm{SL}_{3}(q)$ (which has index two in the full stabilizer, see e.g. [4]). The pointwise stabilizer of that subhexagon is the center of $\mathrm{SL}_{3}(q)$, which is again trivial if 3 divides $q$. More generally, we can now show the following result.

Corollary 5.9 Suppose that $\mathcal{S}$ is a generalized hexagon of order $s$ and $\theta$ is a nontrivial automorphism of $\mathcal{S}$. If $s$ is a multiple of 3 , then $\theta$ cannot fix any thin subhexagon of order $(1, s)$ pointwise.

Proof. Suppose that $\mathcal{S}^{\prime}$ is a thin subhexagon of $\mathcal{S}$ of order $(1, s)$ which is fixed pointwise by $\theta$. Then we claim $f_{0}=2\left(1+s+s^{2}\right)=\left|\mathcal{S}^{\prime}\right|$. Indeed, if $\theta$ fixes a point not belonging to $\mathcal{S}^{\prime}$, then $\theta$ would fix a subhexagon $\mathcal{S}^{\prime \prime}$ of order $\left(s^{\prime \prime}, s\right)$ with $\mathcal{S}^{\prime} \subset \mathcal{S}^{\prime \prime} \subseteq \mathcal{S}$. By [11] it 
follows that $s \geq s^{\prime \prime 2} t$ so $s \geq s^{\prime \prime 2} s$. Hence $s^{\prime \prime}=1$, a contradiction. Now suppose that $x$ is a point of $\mathcal{S}^{\prime}$. There are $s+1$ lines through $x$ in $\mathcal{S}^{\prime}$ and on each of these lines there is precisely one other point which belongs to $\mathcal{S}^{\prime}$. Take such a point $y$. On the line $x y$ of $\mathcal{S}$ there are $s-1$ other points which are mapped to each other (not fixed). So we already have $\left(1+s+s^{2}\right)(s+1)(s-1)$ points which are mapped to a collinear point by $\theta$. Suppose that there is a point $z$ of $\mathcal{S}$ which does not lie on a line of $\mathcal{S}^{\prime}$ and which is mapped to a point collinear to itself. Then by [11] the line $z z^{\theta}$ intersects a line of $\mathcal{S}^{\prime}$ which is fixed under $\theta$. But then the line $z z^{\theta}$ also has to be fixed which leads to a contradiction. Hence $f_{1}=\left(1+s+s^{2}\right)(s+1)(s-1)$. Now suppose that there is a point $u$ which is mapped to a point at distance 4 from itself. There exists a point $u^{\prime}$ which is collinear to $u$ and also to $u^{\theta}$. We have two possibilities, either the point $u^{\prime}$ is incident with a line $L$ of $\mathcal{S}^{\prime}$ or it is not. In the first case the point $u^{\prime}$ should be fixed, because it is the unique point from $L$ collinear to both $u$ and $u^{\theta}$. But it cannot be a point of $\mathcal{S}^{\prime}$ so we obtain a contradiction. In the second case, again by [11] the line $u u^{\prime}$ intersects a line $M$ of $\mathcal{S}^{\prime}$, so the line $u u^{\prime}$ is mapped to a line trough $u^{\theta}$ which intersects the same line $M$ of $\mathcal{S}^{\prime}$. Hence we obtain a quadrangle and we have a contradiction. Consequently $f_{2}=0$. Because of Theorem 5.1 and Theorem 5.4 we have the following equations

$$
\left\{\begin{array}{l}
k_{1} 3 s+k_{2} s+(1+s)^{2}=(1+s) 2\left(1+s+s^{2}\right)+\left(1+s+s^{2}\right)(1+s)(s-1), \\
k_{1} 9 s^{2}+k_{2} s^{2}+(1+s)^{4}=(1+2 s)(1+s) 2\left(1+s+s^{2}\right)+ \\
\quad(1+3 s)\left(1+s+s^{2}\right)(1+s)(s-1) .
\end{array}\right.
$$

Hence

$$
\left\{\begin{array}{l}
k_{1}=\frac{(1+s)\left(1+s+s^{2}\right)}{3} \\
k_{2}=s+s^{2}
\end{array}\right.
$$

We see that $k_{2}$ is an integer, but $k_{1}$ also has to be an integer. It follows that $s$ cannot be a multiple of 3 .

\section{Collineations of octagons and dualities of quadran- gles}

Suppose that $\mathcal{S}$ is a generalized octagon. Let $\theta$ be an automorphism of $\mathcal{S}$ and let $f_{i}$ be the number of points for which $\mathrm{d}\left(x, x^{\theta}\right)=i$ in the point graph. The matrices $M, A$ and $Q$ are defined analogously as before. The eigenvalues of $M$ are as follows (cf. Table 6.4 in $[2])$ : 


\begin{tabular}{|c|c|}
\hline eigenvalues of $M$ & multiplicity \\
\hline 0 & $m_{0}=\frac{s^{4}(1+s t)\left(1+s^{2} t^{2}\right)}{(s+t)\left(s^{2}+t^{2}\right)}$ \\
$(s+1)(t+1)$ & $m_{1}=1$ \\
$s+t+\sqrt{2 s t}$ & $m_{2}=\frac{(1+t) s t(1+s)(1+s t)\left(1+s^{2} t^{2}\right)}{4\left(s(t-1)^{2}+t(s-1)^{2}+2 s t+(s-1)(t-1) \sqrt{2 s t}\right)}$ \\
$s+t-\sqrt{2 s t}$ & $m_{3}=\frac{(1+t) s t(1+s)(1+s t)\left(1+s^{2} t^{2}\right)}{4\left(s(t-1)^{2}+t(s-1)^{2}+2 s t-(s-1)(t-1) \sqrt{2 s t}\right)}$ \\
$s+t$ & $m_{4}=\frac{(1+t) s t(1+s)(1+s t)\left(1+s^{2} t^{2}\right)}{2\left(s(t-1)^{2}+t(s-1)^{2}+4 s t\right)}$ \\
\hline
\end{tabular}

Theorem 6.1 Let $\mathcal{S}$ be a generalized octagon of order $(s, t)$ and let $\theta$ be an automorphism of $\mathcal{S}$. If $f_{0}$ and $f_{1}$ are as before, then for some integers $k_{1}, k_{2}$ and $k_{3}$ there holds

$$
k_{1}(s+t+\sqrt{2 s t})+k_{2}(s+t-\sqrt{2 s t})+k_{3}(s+t)+(1+s)(1+t)=(1+t) f_{0}+f_{1} .
$$

Proof. This proof is totally analogous to the proof of Theorem 5.1.

Lemma 6.2 Let $\mathcal{S}$ be a generalized octagon of order $(s, t)$ and let $\theta$ be an automorphism of $\mathcal{S}$. If $s$ and $t$ are not relatively prime, then there exists at least one fixpoint or at least one point which is mapped to a point collinear to itself.

Proof. This proof is totally analogous to the proof of Corollary 5.2.

Note that in a thick generalized octagon $s$ and $t$ cannot be odd at the same time since $\sqrt{2 s t}$ is an integer and hence either the number of points or the number of lines is odd, or both are. So for a thick generalized octagon, we conclude:

Corollary 6.3 Let $\mathcal{S}$ be a thick generalized octagon of order $(s, t)$ and let $\theta$ be an involution of $\mathcal{S}$. Then there exists at least one fixpoint or at least one fixline.

Proof. By our foregoing observations, either the number of points is odd, or the number of lines is odd. If the number of points is odd, than every involution fixes at least one point. If the number of lines is odd, than every involution fixes at least one line.

If we have a thin octagon, then we obtain: 


$$
k_{1}(1+t+\sqrt{2 t})+k_{2}(1+t-\sqrt{2 t})+k_{3}(1+t)+2(1+t)=(1+t) f_{0}+f_{1}
$$

If $2 t$ is not a square, then it follows that $k_{1}=k_{2}$, hence:

$$
k_{1} 2(1+t)+k_{3}(1+t)+2(1+t)=(1+t) f_{0}+f_{1}
$$

Note that this implies that in a generalized quadrangle of order $t$, with $2 t$ not a square, the number of absolute elements of a duality is divisible by $1+t$. But we will do better in Corollary 6.7.

Theorem 6.4 Let $\mathcal{S}$ be a generalized octagon of order $(s, t)$ and let $\theta$ be an automorphism of $\mathcal{S}$. If $f_{0}, f_{1}$ and $f_{2}$ are as before, then for the integers $k_{1}, k_{2}$ and $k_{3}$ obtained in Theorem 6.1 there holds

$$
\begin{gathered}
k_{1}(s+t+\sqrt{2 s t})^{2}+k_{2}(s+t-\sqrt{2 s t})^{2}+k_{3}(s+t)^{2}+((1+s)(1+t))^{2}= \\
(1+s+t)(1+t) f_{0}+(1+s+2 t) f_{1}+f_{2}
\end{gathered}
$$

Proof. This proof is totally analogous to the proof of Theorem 5.4.

For a thin octagon we obtain:

$k_{1}(1+t+\sqrt{2 t})^{2}+k_{2}(1+t-\sqrt{2 t})^{2}+k_{3}(1+t)^{2}+(2(1+t))^{2}=(2+t)(1+t) f_{0}+(2+2 t) f_{1}+f_{2}$.

If $2 t$ is not a square, then it follows that $k_{1}=k_{2}$ and we obtain:

$$
k_{1} 2\left(1+4 t+t^{2}\right)+k_{3}(1+t)^{2}+(2(1+t))^{2}=(2+t)(1+t) f_{0}+(2+2 t) f_{1}+f_{2} .
$$

Theorem 6.5 Let $\mathcal{S}$ be a generalized octagon of order $(s, t)$ and let $\theta$ be a nontrivial automorphism of $\mathcal{S}$. If $f_{0}, f_{1}, f_{2}$ and $f_{3}$ are defined as above, then for the integers $k_{1}, k_{2}$ and $k_{3}$ of Theorem 6.1 there holds

$$
\begin{gathered}
k_{1}(s+t+\sqrt{2 s t})^{3}+k_{2}(s+t-\sqrt{2 s t})^{3}+k_{3}(s+t)^{3}+((1+s)(1+t))^{3}= \\
\left(s(s-1)(1+t)+3 s(1+t)^{2}+(1+t)^{3}\right) f_{0} \\
+\left(s(1+t)+(s-1)^{2}+s t+3(1+t)(s-1)+3(1+t)^{2}\right) f_{1} \\
+(2(s-1)+3(1+t)) f_{2}+f_{3} .
\end{gathered}
$$


Proof. Suppose that $M, A$ and $Q$ are defined as before. In the same way as in the proof of Theorems 5.1 and 5.4 we can prove that $\operatorname{tr}\left(Q M^{3}\right)=k_{1}(s+t+\sqrt{2 s t})^{3}+k_{2}(s+t-$ $\sqrt{2 s t})^{3}+k_{3}(s+t)^{3}+((1+s)(1+t))^{3}$, with $k_{1}, k_{2}$ and $k_{3}$ integers. On the other hand, because of Lemma 3.3 and the values for $p_{j}^{i}$ given after that lemma, we can calculate that $A^{3}=\left(a_{i j}\right)$ is the matrix with $s(s-1)(1+t)$ along the main diagonal and on the other entries we have $a_{i j}=s(1+t)+(s-1)^{2}+s t$ if $x_{i} \sim x_{j}, a_{i j}=2(s-1)$ if $\mathrm{d}\left(x_{i}, x_{j}\right)=2, a_{i j}=1$ if $\mathrm{d}\left(x_{i}, x_{j}\right)=3$ and $a_{i j}=0$ otherwise. Hence $\operatorname{tr}\left(Q A^{3}\right)=$ $s(s-1)(1+t) f_{0}+\left(s(1+t)+(s-1)^{2}+s t\right) f_{1}+2(s-1) f_{2}+f_{3}$. Because of the proof of Theorem 5.4 we know that $\operatorname{tr}\left(Q A^{2}\right)=s(1+t) f_{0}+(s-1) f_{1}+f_{2}, \operatorname{tr}(Q A)=f_{1}$ and $\operatorname{tr}(Q)=f_{0}$. Hence

$$
\begin{aligned}
& \operatorname{tr}\left(Q M^{3}\right) \\
= & \operatorname{tr}\left(Q(A+(1+t) I)^{3}\right) \\
= & \operatorname{tr}\left(Q A^{3}\right)+3(1+t) \operatorname{tr}\left(Q A^{2}\right)+3(1+t)^{2} \operatorname{tr}(Q A)+(1+t)^{3} \operatorname{tr}(Q) \\
= & s(s-1)(1+t) f_{0}+\left(s(1+t)+(s-1)^{2}+s t\right) f_{1}+2(s-1) f_{2}+f_{3} \\
& +3(1+t)\left(s(1+t) f_{0}+(s-1) f_{1}+f_{2}\right)+3(1+t)^{2} f_{1}+(1+t)^{3} f_{0} \\
= & \left(s(s-1)(1+t)+3 s(1+t)^{2}+(1+t)^{3}\right) f_{0} \\
& +\left(s(1+t)+(s-1)^{2}+s t+3(1+t)(s-1)+3(1+t)^{2}\right) f_{1} \\
& +(2(s-1)+3(1+t)) f_{2}+f_{3} .
\end{aligned}
$$

Using Lemma 3.2 as before, the proof of the theorem is complete.

For a thin octagon with $s=1$ we obtain:

$$
\begin{gathered}
k_{1}(1+t+\sqrt{2 t})^{3}+k_{2}(1+t-\sqrt{2 t})^{3}+k_{3}(1+t)^{3}+(2(1+t))^{3}= \\
\left(3(1+t)^{2}+(1+t)^{3}\right) f_{0}+\left(1+2 t+3(1+t)^{2}\right) f_{1}+3(1+t) f_{2}+f_{3} .
\end{gathered}
$$

If $2 t$ is not a square, then it follows that $k_{1}=k_{2}$ and we obtain:

$$
\begin{gathered}
k_{1} 2\left(1+9 t+9 t^{2}+t^{3}\right)+k_{3}(1+t)^{3}+(2(1+t))^{3}= \\
\left(3(1+t)^{2}+(1+t)^{3}\right) f_{0}+\left(1+2 t+3(1+t)^{2}\right) f_{1}+3(1+t) f_{2}+f_{3} .
\end{gathered}
$$

Corollary 6.6 Suppose that we have a thin octagon of order $(1, t)$. Consider a duality in the underlying generalized quadrangle. If $2 t$ is not a square, then $f_{1}=2(1+t)$. If $2 t$ is a square, then $f_{1} \equiv 2 \bmod 2 \sqrt{2} t$. In particular there is at least one absolute point and one absolute line. 
Proof. Since we have a duality in the underlying generalized quadrangle, we know that $f_{0}=0$ and $f_{2}=0$. Because of Lemma 3.2, Theorems 6.1, 6.4 and 6.5, we have the following equations:

$$
\left\{\begin{array}{l}
k_{1}(1+t+\sqrt{2 t})+k_{2}(1+t-\sqrt{2 t})+k_{3}(1+t)+2(1+t)=f_{1}, \\
k_{1}(1+t+\sqrt{2 t})^{2}+k_{2}(1+t-\sqrt{2 t})^{2}+k_{3}(1+t)^{2}+(2(1+t))^{2}=(2+2 t) f_{1} \\
k_{1}(1+t+\sqrt{2 t})^{3}+k_{2}(1+t-\sqrt{2 t})^{3}+k_{3}(1+t)^{3}+(2(1+t))^{3}= \\
\quad\left(1+2 t+3(1+t)^{2}\right) f_{1}+f_{3} .
\end{array}\right.
$$

Because $f_{0}$ and $f_{2}$ are 0 , we know that $f_{1}+f_{3}=2(1+t)\left(1+t^{2}\right)$. Hence:

$$
\left\{\begin{array}{l}
k_{1}=\frac{\sqrt{2}\left(-2 t+f_{1}-2\right)}{4 \sqrt{t}} \\
k_{2}=-\frac{\sqrt{2}\left(-2 t+f_{1}-2\right)}{4 \sqrt{t}} \\
k_{3}=0 \\
f_{3}=2\left(t^{3}+t^{2}+t+1\right)-f_{1}
\end{array}\right.
$$

So $\frac{\sqrt{2}\left(-2 t+f_{1}-2\right)}{4 \sqrt{t}}$ has to be an integer. In the case that $2 t$ is not a square, this only holds if $-2 t+f_{1}-2=0$. Hence $f_{1}=2(1+t)$ if $2 t$ is not a square. If $2 t$ is a square, then $f_{1}-2$ has to be a multiple of $2 \sqrt{2 t}$. Hence $f_{1} \equiv 2 \bmod 2 \sqrt{2 t}$.

From Lemma 3.4 now immediately follows.

Corollary 6.7 Suppose that $\theta$ is a duality of a generalized quadrangle of order $t$. If $2 t$ is not a square, then it has $1+t$ absolute points and $1+t$ absolute lines, and there are $(1+t) t^{2}$ points which are mapped to a line at distance 3 and $(1+t) t^{2}$ lines which are mapped to a point at distance 3 . If $2 t$ is a perfect square, then it has $1 \bmod \sqrt{2 t}$ absolute points and equally many absolute lines.

If $2 t$ is a square, then one can again construct examples of dualities in a generalized quadrangle of order $t$, namely in the symplectic quadrangle $W(t)$, admitting the lower bound 1 of absolute points given in the previous corollary. Indeed, consider a polarity $\rho$ and compose it with a nontrivial central root elation $\tau$ whose center is an absolute point $x$. The resulting duality $\theta=\rho \tau$ has $x$ as unique absolute point. Indeed, suppose by way of contradiction that the point $y \neq x$ is absolute for $\theta$. Since $\tau$ is involutive, this implies that $y^{\rho} \mathrm{I} y^{\tau}$. It is easy to see that $x$ and $y$ are not collinear and that $x y^{\rho}$. Since $\tau$ is central, the unique point $z$ on $y^{\rho}$ collinear with $x$ is also collinear with $y$. Applying $\rho$ to the chain $y \mathrm{I} y z \mathrm{I} z \mathrm{I} y^{\rho}$, we deduce that $z^{\rho}=y z$, hence $z$ is an absolute point for $\rho$, contradicting the fact that $z$ is collinear with $x$. 


\section{Collineations of dodecagons and dualities of hexagons}

Suppose that $\mathcal{S}$ is a generalized dodecagon. Let $\theta$ be an automorphism of $\mathcal{S}$ and let $f_{i}$ be the number of points for which $\mathrm{d}\left(x, x^{\theta}\right)=i$ in the point graph, with $i \in\{0,1,2,3,4,5\}$. The matrices $M, A$ and $Q$ are defined analogously as before. The eigenvalues of $M$ are as follows (cf. [5]):

\begin{tabular}{|c|c|}
\hline eigenvalues of $M$ & multiplicity \\
\hline 0 & $m_{0}$ \\
$(s+1)(t+1)$ & $m_{1}=1$ \\
$s+t+\sqrt{s t}$ & $m_{2}$ \\
$s+t-\sqrt{s t}$ & $m_{3}$ \\
$s+t+\sqrt{3 s t}$ & $m_{4}$ \\
$s+t-\sqrt{3 s t}$ & $m_{5}$ \\
$s+t$ & $m_{6}$ \\
\hline
\end{tabular}

Thick finite generalized dodecagons do not exist, but nevertheless we formulate the following results with general $s$ and $t$. In real life, either $s$ or $t$ is equal to 1 , but the formulae do not seem to be equivalent. Afterwards, we apply our results to the case $s=1$, implying results for dualities of generalized hexagons.

Theorem 7.1 Let $\mathcal{S}$ be a generalized dodecagon of order $(s, t)$ and let $\theta$ be an automorphism of $\mathcal{S}$. If $f_{0}$ is the number of points fixed by $\theta$ and $f_{1}$ is the number of points $x$ for which $x \sim x^{\theta}$, then for some integers $k_{1}, k_{2}, k_{3}, k_{4}$ and $k_{5}$ there holds

$k_{1}(s+t+\sqrt{s t})+k_{2}(s+t-\sqrt{s t})+k_{3}(s+t+\sqrt{3 s t})+k_{4}(s+t-\sqrt{3 s t})+k_{5}(s+t)+(1+s)(1+t)=$ $(1+t) f_{0}+f_{1}$.

Proof. This proof is totally analogous to the proof of Theorem 5.1. 
Theorem 7.2 Let $\mathcal{S}$ be a generalized dodecagon of order $(s, t)$ and let $\theta$ be an automorphism of $\mathcal{S}$. If $f_{i}, i=0,1,2$, is defined as above, then for the integers $k_{1}, k_{2}, k_{3}, k_{4}$ and $k_{5}$ obtained in Theorem 7.1 there holds

$$
\begin{gathered}
k_{1}(s+t+\sqrt{s t})^{2}+k_{2}(s+t-\sqrt{s t})^{2}+k_{3}(s+t+\sqrt{3 s t})^{2}+k_{4}(s+t-\sqrt{3 s t})^{2}+k_{5}(s+t)^{2}+ \\
((1+s)(1+t))^{2}=(1+s+t)(1+t) f_{0}+(1+s+2 t) f_{1}+f_{2} .
\end{gathered}
$$

Proof. This proof is totally analogous to the proof of Theorem 5.4.

Theorem 7.3 Let $\mathcal{S}$ be a generalized dodecagon of order $(s, t)$ and let $\theta$ be an automorphism of $\mathcal{S}$. If $f_{i}, i=0,1,2,3$, is as before, then for the integers $k_{1}, k_{2}, k_{3}, k_{4}$ and $k_{5}$ of Theorem 7.1 there holds

$$
\begin{gathered}
k_{1}(s+t+\sqrt{s t})^{3}+k_{2}(s+t-\sqrt{s t})^{3}+k_{3}(s+t+\sqrt{3 s t})^{3}+k_{4}(s+t-\sqrt{3 s t})^{3}+k_{5}(s+t)^{3}+ \\
((1+s)(1+t))^{3}= \\
\left(s(s-1)(1+t)+3 s(1+t)^{2}+(1+t)^{3}\right) f_{0} \\
+\left(s(1+t)+(s-1)^{2}+s t+3(1+t)(s-1)+3(1+t)^{2}\right) f_{1} \\
+(2(s-1)+3(1+t)) f_{2}+f_{3} .
\end{gathered}
$$

Proof. This proof is totally analogous to the proof of Theorem 6.5.

Theorem 7.4 Let $\mathcal{S}$ be a generalized dodecagon of order $(s, t)$ and let $\theta$ be an automorphism of $\mathcal{S}$. If $f_{i}, i=0,1,2,3,4$, is as before, then for the integers $k_{1}, k_{2}, k_{3}, k_{4}$ and $k_{5}$ of Theorem 7.1 there holds

$$
\begin{gathered}
k_{1}(s+t+\sqrt{s t})^{4}+k_{2}(s+t-\sqrt{s t})^{4}+k_{3}(s+t+\sqrt{3 s t})^{4}+k_{4}(s+t-\sqrt{3 s t})^{4}+k_{5}(s+t)^{4}+ \\
((1+s)(1+t))^{4}= \\
\left(\left(s(1+t)+(s-1)^{2}+s t\right)(1+t) s+4 s(s-1)(1+t)^{2}+6 s(1+t)^{3}+(1+t)^{4}\right) f_{0} \\
+\left(s(s-1)(1+t)+(s-1)\left(s(1+t)+(s-1)^{2}+s t\right)+2(s-1) s t+4(1+t)(s(1+t)\right. \\
\left.\left.+(s-1)^{2}+s t\right)+6(1+t)^{2}(s-1)+4(1+t)^{3}\right) f_{1} \\
+\left(s(1+t)+3(s-1)^{2}+2 s t+8(1+t)(s-1)+6(1+t)^{2}\right) f_{2} \\
+(3(s-1)+4(1+t)) f_{3}+f_{4} .
\end{gathered}
$$

Proof. Suppose that $M, A$ and $Q$ are defined as before. In the same way as in the proof of Theorems 5.1, 5.4 and 6.5 we can prove that $\operatorname{tr}\left(Q M^{4}\right)=k_{1}(s+t+\sqrt{s t})^{4}+$ $k_{2}(s+t-\sqrt{s t})^{4}+k_{3}(s+t+\sqrt{3 s t})^{4}+k_{4}(s+t-\sqrt{3 s t})^{4}+k_{5}(s+t)^{4}+((1+s)(1+t))^{4}$, with $k_{1}, k_{2}, k_{3}, k_{4}$ and $k_{5}$ integers. On the other hand, because of Lemma 3.3 and the values for $p_{j}^{i}$ given after that lemma, we can calculate that $A^{4}=\left(a_{i j}\right)$ is the matrix with $\left(s(1+t)+(s-1)^{2}+s t\right)(1+t) s$ on the main diagonal while on the other entries we have 


$$
\begin{array}{ll}
a_{i j}=s(s-1)(1+t)+(s-1)\left(s(1+t)+(s-1)^{2}+s t\right)+2(s-1) s t & \text { if } x_{i} \sim x_{j} \\
a_{i j}=s(1+t)+3(s-1)^{2}+2 s t & \text { if } \mathrm{d}\left(x_{i}, x_{j}\right)=2 \\
a_{i j}=3(s-1) & \text { if } \mathrm{d}\left(x_{i}, x_{j}\right)=3 \\
1 & \text { if } \mathrm{d}\left(x_{i}, x_{j}\right)=4 \\
0 & \text { otherwise. }
\end{array}
$$

Hence

$$
\begin{aligned}
& \operatorname{tr}\left(Q A^{4}\right)=\left(\left(s(1+t)+(s-1)^{2}+s t\right)(1+t) s\right) f_{0} \\
& \quad+\left(s(s-1)(1+t)+(s-1)\left(s(1+t)+(s-1)^{2}+s t\right)+2(s-1) s t\right) f_{1} \\
& \quad+\left(s(1+t)+3(s-1)^{2}+2 s t\right) f_{2}+3(s-1) f_{3}+f_{4} .
\end{aligned}
$$

The rest of the proof is totally analogous to the proof of Theorem 6.5.

Theorem 7.5 Let $\mathcal{S}$ be a generalized dodecagon of order $(s, t)$ and let $\theta$ be an automorphism of $\mathcal{S}$. If $f_{i}, i=0,1,2,3,4,5$, is as before, then for the integers $k_{1}, k_{2}, k_{3}, k_{4}$ and $k_{5}$ of Theorem 7.1 there holds

$$
\begin{gathered}
k_{1}(s+t+\sqrt{s t})^{5}+k_{2}(s+t-\sqrt{s t})^{5}+k_{3}(s+t+\sqrt{3 s t})^{5}+k_{4}(s+t-\sqrt{3 s t})^{5}+k_{5}(s+t)^{5}+ \\
((1+s)(1+t))^{5}=\left(s(t+1)\left(s(s-1)(1+t)+(s-1)\left(s(1+t)+(s-1)^{2}+s t\right)+2(s-1) s t\right)+\right. \\
\left.5(1+t)\left(s(1+t)+(s-1)^{2}+s t\right)(1+t) s+10(1+t)^{2} s(s-1)(1+t)+10(1+t)^{3} s(t+1)+(1+t)^{5}\right) f_{0} \\
+\left(\left(s(1+t)+(s-1)^{2}+s t\right)(1+t) s+(s-1)\left(s(s-1)(1+t)+(s-1)\left(s(1+t)+(s-1)^{2}+s t\right)+\right.\right. \\
2(s-1) s t)+s t\left(s(1+t)+3(s-1)^{2}+2 s t\right)+5(1+t)(s(s-1)(1+t)+(s-1)(s(1+t)+ \\
\left.\left.(s-1)^{2}+s t\right)+2(s-1) s t\right)+10(1+t)^{2}\left(s(1+t)+(s-1)^{2}+s t\right)+10(1+t)^{3}(s-1)+ \\
\left.5(1+t)^{4}\right) f_{1} \\
+\left(s(s-1)(1+t)+(s-1)\left(s(1+t)+(s-1)^{2}+s t\right)+2(s-1) s t+(s-1)\left(s(1+t)+3(s-1)^{2}+2 s t\right)\right. \\
\left.+3 s t(s-1)+5(1+t)\left(s(1+t)+3(s-1)^{2}+2 s t\right)+10(1+t)^{2} 2(s-1)+10(1+t)^{3}\right) f_{2} \\
\left(s(1+t)+3(s-1)^{2}+2 s t+3(s-1)^{2}+s t+5(t+1) 3(s-1)+10(t+1)^{2}\right) f_{3} \\
+(4(s-1)+5(t+1)) f_{4}+f_{5} .
\end{gathered}
$$

Proof. Suppose that $M, A$ and $Q$ are defined as before. In the same way as in the proof of Theorems 5.1, 5.4 and 6.5 we can prove that $\operatorname{tr}\left(Q M^{5}\right)=k_{1}(s+t+\sqrt{s t})^{5}+k_{2}(s+t-$ $\sqrt{s t})^{5}+k_{3}(s+t+\sqrt{3 s t})^{5}+k_{4}(s+t-\sqrt{3 s t})^{5}+k_{5}(s+t)^{5}+((1+s)(1+t))^{5}$, with $k_{1}, k_{2}$, $k_{3}, k_{4}$ and $k_{5}$ the integers of Theorem 7.1 (by Lemma 3.2). On the other hand, because of Lemma 3.3 and the values for $p_{j}^{i}$ given after that lemma, we can calculate that $A^{4}=\left(a_{i j}\right)$ is the matrix with $s(t+1)\left(s(s-1)(1+t)+(s-1)\left(s(1+t)+(s-1)^{2}+s t\right)+2(s-1) s t\right)$ on the main diagonal while on the other entries we have 


$$
\begin{array}{rlrl}
a_{i j}= & \left(s(1+t)+(s-1)^{2}+s t\right)(1+t) s+(s-1)(s(s-1)(1+t) & & \\
& \left.+(s-1)\left(s(1+t)+(s-1)^{2}+s t\right)+2(s-1) s t\right) & & \\
& +s t\left(s(1+t)+3(s-1)^{2}+2 s t\right) & & \text { if } x_{i} \sim x_{j} \\
a_{i j}= & s(s-1)(1+t)+(s-1)\left(s(1+t)+(s-1)^{2}+s t\right)+2(s-1) s t & & \\
& +(s-1)\left(s(1+t)+3(s-1)^{2}+2 s t\right)+3 s t(s-1) & & \text { if } \mathrm{d}\left(x_{i}, x_{j}\right)=2 \\
a_{i j}= & s(1+t)+3(s-1)^{2}+2 s t+3(s-1)^{2}+s t & & \text { if } \mathrm{d}\left(x_{i}, x_{j}\right)=3 \\
a_{i j}= & 4(s-1) & & \text { if } \mathrm{d}\left(x_{i}, x_{j}\right)=4 \\
1 & & & \text { if d }\left(x_{i}, x_{j}\right)=5 \\
0 & & \text { otherwise. }
\end{array}
$$

Hence

$$
\begin{aligned}
& \operatorname{tr}\left(Q A^{5}\right)=\left(s(t+1)\left(s(s-1)(1+t)+(s-1)\left(s(1+t)+(s-1)^{2}+s t\right)+2(s-1) s t\right)\right) f_{0} \\
& \quad+\left(\left(s(1+t)+(s-1)^{2}+s t\right)(1+t) s+(s-1)(s(s-1)(1+t)+(s-1)(s(1+t)\right. \\
& \left.\left.\left.\quad+(s-1)^{2}+s t\right)+2(s-1) s t\right)+s t\left(s(1+t)+3(s-1)^{2}+2 s t\right)\right) f_{1} \\
& \quad+\left(s(s-1)(1+t)+(s-1)\left(s(1+t)+(s-1)^{2}+s t\right)+2(s-1) s t\right. \\
& \left.\quad+(s-1)\left(s(1+t)+3(s-1)^{2}+2 s t\right)+3 s t(s-1)\right) f_{2} \\
& \quad+\left(s(1+t)+3(s-1)^{2}+2 s t+3(s-1)^{2}+s t\right) f_{3}+4(s-1) f_{4}+f_{5} .
\end{aligned}
$$

We have

$$
\begin{aligned}
& \operatorname{tr}\left(Q M^{5}\right) \\
= & \operatorname{tr}\left(Q(A+(1+t) I)^{5}\right) \\
= & \operatorname{tr}\left(Q A^{5}\right)+5(1+t) \operatorname{tr}\left(Q A^{4}\right)+10(1+t)^{2} \operatorname{tr}\left(Q A^{3}\right)+10(1+t)^{3} \operatorname{tr}\left(Q A^{2}\right) \\
& +5(1+t)^{4} \operatorname{tr}(Q A)+(1+t)^{5} \operatorname{tr}(Q) .
\end{aligned}
$$

If we substitute the formula for $\operatorname{tr}\left(Q A^{5}\right)$ which we obtained above and the formulas which we obtained in Theorems 5.1, 5.4, 6.5 and 7.4 for $\operatorname{tr}\left(Q A^{4}\right), \operatorname{tr}\left(Q A^{3}\right), \operatorname{tr}\left(Q A^{2}\right), \operatorname{tr}(Q A)$ and $\operatorname{tr}(Q)$, then we obtain the assertion.

Corollary 7.6 Suppose that we have a thin dodecagon of order $(1, t)$. Consider a duality in the underlying generalized hexagon. If $3 t$ and $t$ are no squares, then $f_{1}=2(1+t)$, $f_{3}=2\left(t^{2}+t^{3}\right)$ and $f_{5}=2\left(t^{4}+t^{5}\right)$. If $3 t$ is a square and $t$ is not a square, then $f_{1} \equiv 2$ $\bmod 2 \sqrt{3 t}$ and $f_{3} \equiv 0 \bmod 2 \sqrt{3 t}$. If $t$ is a square and $3 t$ is not a square, then $f_{1} \equiv 2$ $\bmod 2 \sqrt{t}$ and $f_{3} \equiv 0 \bmod 2 \sqrt{t}$. In particular there is always at least one absolute point and one absolute line. 
Proof. Since we have a duality in the underlying generalized hexagon, we know that $f_{0}=0, f_{2}=0$ and $f_{4}=0$. Because of Theorems 7.1, 7.2, 7.3, 7.4 and 7.5, we have the following equations:

$$
\left\{\begin{array}{c}
k_{1}(1+t+\sqrt{t})+k_{2}(1+t-\sqrt{t})+k_{3}(1+t+\sqrt{3 t})+k_{4}(1+t-\sqrt{3 t})+k_{5}(1+t) \\
\quad+2(1+t)=f_{1} \\
k_{1}(1+t+\sqrt{t})^{2}+k_{2}(1+t-\sqrt{t})^{2}+k_{3}(1+t+\sqrt{3 t})^{2}+k_{4}(1+t-\sqrt{3 t})^{2}+k_{5}(1+t)^{2} \\
\quad+(2(1+t))^{2}=(2+2 t) f_{1}, \\
k_{1}(1+t+\sqrt{t})^{3}+k_{2}(1+t-\sqrt{t})^{3}+k_{3}(1+t+\sqrt{3 t})^{3}+k_{4}(1+t-\sqrt{3 t})^{3}+k_{5}(1+t)^{3} \\
\quad+(2(1+t))^{3}=\left(1+2 t+3(1+t)^{2}\right) f_{1}+f_{3}, \\
k_{1}(1+t+\sqrt{t})^{4}+k_{2}(1+t-\sqrt{t})^{4}+k_{3}(1+t+\sqrt{3 t})^{4}+k_{4}(1+t-\sqrt{3 t})^{4}+k_{5}(1+t)^{4} \\
\quad+(2(1+t))^{4}=\left(4(1+t)(1+2 t)+4(1+t)^{3}\right) f_{1}+4(1+t) f_{3}, \\
k_{1}(1+t+\sqrt{t})^{5}+k_{2}(1+t-\sqrt{t})^{5}+k_{3}(1+t+\sqrt{3 t})^{5}+k_{4}(1+t-\sqrt{3 t})^{5}+k_{5}(t+1)^{5} \\
\quad+(2(t+1))^{5}=\left(5 t^{4}+40 t^{3}+85 t^{2}+64 t+16\right) f_{1}+\left(10 t^{2}+24 t+11\right) f_{3}+f_{5} .
\end{array}\right.
$$

Because $f_{0}, f_{2}$ and $f_{4}$ are 0 , we know that $f_{1}+f_{3}+f_{5}=\frac{2\left(t^{6}-1\right)}{t-1}$. Hence:

$$
\left\{\begin{aligned}
k_{1} & =-\frac{-2 t^{3}-f_{1} t+f_{3}+f_{1}-2}{4 \sqrt{t^{3}}} \\
k_{2} & =\frac{-2 t^{3}-f_{1} t+f_{3}+f_{1}-2}{4 \sqrt{t^{3}}} \\
k_{3} & =\frac{\left(-2 t^{3}-4 t^{2}-4 t-2+f_{1} t+f_{3}+f_{1}\right) \sqrt{3}}{12 \sqrt{t^{3}}} \\
k_{4} & =-\frac{\left(-2 t^{3}-4 t^{2}-4 t-2+f_{1} t+f_{3}+f_{1}\right) \sqrt{3}}{12 \sqrt{t^{3}}} \\
k_{5} & =0 \\
f_{5} & =2\left(t^{5}+t^{4}+t^{3}+t^{2}+t+1\right)-f_{1}-f_{3} .
\end{aligned}\right.
$$

So $\frac{-2 t^{3}-f_{1} t+f_{3}+f_{1}-2}{4 \sqrt{t^{3}}}$ and $\frac{\left(-2 t^{3}-4 t^{2}+f_{1} t-2-4 t+f_{3}+f_{1}\right) \sqrt{3}}{12 \sqrt{t^{3}}}$ have to be integers. In the case that $3 t$ and $t$ are no squares, this only holds if $-2 t^{3}-f_{1} t+f_{3}+f_{1}-2=0$ and $-2 t^{3}-4 t^{2}-$ $4 t-2+f_{1} t+f_{3}+f_{1}=0$. Hence $f_{1}=2(1+t)$ and $f_{3}=2\left(t^{2}+t^{3}\right)$ if $3 t$ and $t$ are no squares. If $3 t$ is a square and $t$ is no square, then $-2 t^{3}-f_{1} t+f_{3}+f_{1}-2=0$ and $-2 t^{3}-4 t^{2}+f_{1} t-2-4 t+f_{3}+f_{1}$ has to be a multiple of $4 t \sqrt{3 t}$. Combining these, we see that $f_{1}-2$ has to be a multiple of $2 \sqrt{3 t}$, which means that $f_{1} \equiv 2 \bmod 2 \sqrt{3 t}$. Substituting this in the former equality yields $f_{3} \equiv 0 \bmod 2 \sqrt{3 t}$. On the other hand, if $t$ is a square and $3 t$ is no square, then $-2 t^{3}-4 t^{2}+f_{1} t-2-4 t+f_{3}+f_{1}=0$ and $-2 t^{3}-f_{1} t+f_{3}+f_{1}-2$ has to be a multiple of $4 t \sqrt{t}$. Hence $f_{1}-2$ has to be a multiple of $2 \sqrt{t}$, which means that $f_{1} \equiv 2 \bmod 2 \sqrt{t}$. Similarly as above, $f_{3} \equiv 0 \bmod 2 \sqrt{t}$.

This immediately implies, in view of Lemma 3.4, the following corollary. 
Corollary 7.7 Suppose that $\theta$ is a duality of a generalized hexagon of order $t$. If none of $3 t$ and $t$ are perfect squares, then $\theta$ has $1+t$ absolute points and $1+t$ absolute lines, there are $t^{2}+t^{3}$ points which are mapped to a line at distance 3 and $t^{2}+t^{3}$ lines which are mapped to a point at distance 3 , and there are $t^{4}+t^{5}$ points which are mapped to a line at distance 5 and $t^{4}+t^{5}$ lines which are mapped to a point at distance 5 . If $t$ is a perfect square, then there are $1 \bmod \sqrt{t}$ absolute points and equally many absolute lines; the number of point mapped onto a line at distance 3 in the incidence graph is divisible by $\sqrt{t}$. If $3 t$ is a perfect square, then there are $1 \bmod \sqrt{3 t}$ absolute points and equally many absolute lines; the number of point mapped onto a line at distance 3 in the incidence graph is divisible by $\sqrt{3 t}$.

We currently do not know of any finite self-dual generalized hexagon of order $t$, with neither $t$ nor $3 t$ a perfect square. If $3 t$ is a square, then similarly as for symplectic quadrangles of order $s$, with $2 s$ a square, one can easily construct dualities of the split Cayley hexagon $\mathrm{H}(t)$ with exactly one absolute point as the composition of a polarity with a nontrivial central collineation with center one of the absolute points of the polarity. If $t$ is a square, then our conjecture is that the number of absolute points of a duality is also exactly $1+t$.

\section{References}

[1] C. T. Benson, On the structure of generalized quadrangles, J. Algebra 15 (1970), $443-454$.

[2] A. E. Brouwer, A. M. Cohen \& A. Neumaier, Distance-Regular Graphs, Ergeb. Math. Grenzgeb. (3) 18, Springer-Verlag, Berlin, 1989.

[3] P. J. Cameron, J. A. Thas \& S. E. Payne, Polarities of generalized hexagons and perfect codes, Geom. Dedicata 5 (1976), 525-528.

[4] J. De Kaey \& H. Van Maldeghem, A characterization of the split Cayley generalized hexagon $\mathbf{H}(q)$ using one subhexagon of order $(1, q)$, Discr. Math. 294 (2005), 109118.

[5] A. De Wispelaere and H. Van Maldeghem, Regular partitions of (weak) finite generalized polygons, to be published in Designs, Codes and Crypt., 26pp. 
[6] W. Feit \& D. Higman, The nonexistence of certain generalized polygons, J. Algebra 1 (1964), 114-131.

[7] J. B. Fraleigh, A First Course in Abstract Algebra, Addison- Wesley, 1994.

[8] A. Offer, On the order of a generalized hexagon admitting an ovoid or spread, $J$. Combin. Theory Ser. A 97 (2002), 184-186.

[9] S. E. Payne \& J. A. Thas, Finite Generalized Quadrangles, Research Notes in Mathematics 110, Pitman Advanced Publishing Program, Boston/London/Melbourne, 1984 .

[10] C. Schneider \& H. Van Maldeghem, Primitive flag-transitive generalized hexagons and octagons, submitted

[11] J. A. Thas, A restriction on the parameters of a subhexagon, J. Combin. Theory Ser. A 21 (1976), 115-117. (35, 38)

[12] J. A. Thas, Generalized polygons, in Handbook of Incidence Geometry, Buildings and Foundations, (ed. F. Buekenhout), Chapter 9, North-Holland, 383-431.

[13] J. Tits, Sur la trialité et certains groupes qui s'en déduisent, Inst. Hautes Études Sci. Publ. Math. 2 (1959), 13-60.

[14] J. Tits, Classification of buildings of spherical type and Moufang polygons: a survey, in "Coll. Intern. Teorie Combin. Acc. Naz. Lincei", Proceedings Roma 1973, Atti dei convegni Lincei 17 (1976), 229-246.

[15] H. Van Maldeghem, Generalized Polygons, Birkhäuser Verlag, Basel, Boston, Berlin, Monographs in Mathematics 93, 1998. 